\title{
FIRST STEPS OF CRACK INITIATION AND PROPAGATION IN FATIGUE OF FCC CRYSTALS STUDIED BY DISLOCATION DYNAMICS
}

\author{
C. DEPRES \\ Laboratoire SYMME. Université Savoie Mt Blanc \\ 74940 ANNECY-LE-VIEUX, FRANCE \\ christophe.depres@univ-savoie.fr \\ G.V. Prasad Reddy \\ Mechanical Metallurgy Division, Indira Gandhi Center for Atomic \\ Research, Kalpakkam, 603102 Tamil Nadu, India \\ C. Robertson \\ Service de Recherche de Métallurgie Appliquée, CEA Saclay, \\ 91191 Gif-sur-Yvette, Paris, France \\ M.Fivel \\ SIMAP-GPM2, Université Grenoble Alpes-CNRS, F-38000 \\ Grenoble, France
}

3D discrete dislocation dynamic (DDD) simulations are performed to simulate stage-I fatigue crack initiation and propagation along the surface, in the primary grain in 316L stainless steel. It emphasizes the effect of the cyclic plasticity on the specificity of the dislocations microstructure and its direct consequence on the surface relief. It shows how this relief favors crack initiation, and how the dislocations microstructure favors its propagation in the primary grain. The evolution of the dislocation structure ahead of crack tip is discussed, and in addition crack tip sliding displacement is estimated for different position of the tip in the grain. A propagation law is given and compared with the initiation law, to highlight the effect of the grain size and the plastic strain on both regimes. It is clearly shown than small grains disfavors crack initiation, but favors crack propagation. An energetical approach is performed to estimate the most probable propagation speed, via the estimation of the maximum decohesion length allowed by the principle of thermodynamics. 